AMERICAN CONGO 



\section{AMERICAN CONGO}

The African American

Freedom Struggle in the Delta

Nan Elizabeth Woodruff

HARVARD UNIVERSITY PRESS

Cambridge, Massachusetts, and London, England 2003 


\section{For Irene Silverblatt}

Copyright (C) 2003 by the President and Fellows of Harvard College All rights reserved

Printed in the United States of America

\section{Library of Congress Cataloging-in-Publication Data}

Woodruff, Nan Elizabeth, 1949-

American Congo : the African American freedom struggle in the Delta / Nan Elizabeth Woodruff.

$$
\text { p. cm. }
$$

Includes bibliographical references and index.

ISBN 0-674-01047-7

1. African Americans-Civil rights-Mississippi-Delta (Region)— History-20th century. 2. African Americans-Civil rights-ArkansasArkansas Delta-History—20th century. 3. Sharecroppers-MississippiDelta (Region)-Social conditions-20th century. 4. SharecroppersArkansas-Arkansas Delta—Social conditions-20th century. 5. Civil rights movements—Mississippi-Delta (Region) — History—20th century. 6. Civil rights movements-Arkansas-Arkansas Delta—History—20th century. 7. Plantation life-Mississippi-Delta (Region)—History-20th century. 8. Plantation life-Arkansas-Arkansas Delta-History-20th century. 9. Delta (Miss. : Region)—Race relations. 10. Arkansas Delta (Ark.)—Race relations. I. Title.

F347.M6W667 2003

976.2'400496073-dc21 